\title{
THE EFFECT OF NOREPINEPHRINE ON VISUAL CORTICAL NEURONS IN KITTENS AND ADULT CATS ${ }^{1}$
}

\author{
T. O. VIDEEN, ${ }^{2}$ N. W. DAW, AND R. K. RADER \\ Department of Physiology and Biophysics and McDonnell Center for Study of Higher Brain Functions, Washington University \\ School of Medicine, St. Louis, Missouri 63110
}

Received August 24, 1983; Revised January 16, 1984; Accepted January 16, 1984

\begin{abstract}
The responses of visual cortical neurons during iontophoresis of norepinephrine (NE) were compared in kittens and adult cats. One type of neuronal "plasticity" (the ability of visual cortical neurons to have their response properties markedly altered during exposure to a restricted visual environment) normally occurs only in young kittens and has been hypothesized to depend on the presence of NE. We investigated this hypothesis in terms of a model of synaptic plasticity which suggested that NE would selectively enhance the evoked responses of neurons and that this enhancement might exist to a greater extent in kittens than in adult cats.

The responses of $72 \%$ ( 43 of 60 ) of all neurons sampled decreased during NE iontophoresis, while the responses of only a few neurons ( 3 of 60 ) increased. Reduced activity occurred in roughly equal percentages of simple and complex cells. We also looked for differences in NE modulation of the "signal-to-noise" ratio of neurons. Most neurons had little spontaneous activity, but, of those that did, the signal-to-noise ratio increased in some instances and decreased in others. No difference in any of the effects of NE was observed between kittens and adult cats. The role of NE in cortical plasticity is discussed.
\end{abstract}

The response properties of visual cortical neurons can be significantly altered by depriving young kittens of normal visual experience (Wiesel and Hubel, 1963; summaries in Movshon and Van Sluyters, 1981; Sherman and Spear, 1982). It has been hypothesized that norepinephrine (NE) is crucially involved in allowing these changes in response properties to occur since depletion of cortical NE by infusing 6-hydroxydopamine (6OHDA) into the cortex or ventricles either prevents or reduces the amount of change (Kasamatsu and Pettigrew, 1976; 1979; Bear et al., 1983; Daw et al., 1983a). Moreover, local microperfusion of NE into the visual cortex of kittens which have first had their visual cortices depleted of catecholamines by treatment with 6-OHDA restores the ability of neuronal response properties to change during visual deprivation (Pettigrew and Kasamatsu, 1978; Kasamatsu et al., 1979). Furthermore, the restoration of normal neuronal response properties after a period of visual deprivation may be accelerated by local perfusion with NE (Kasamatsu et al., 1981).

\footnotetext{
${ }^{1}$ This work was supported by National Institutes of Health Research Grant EY00053 and Program Project Grant NS15070. T. O. V. was supported by National Institutes of Health Training Grant EY07057 and by the McDonnell Center for Studies of Higher Brain Function.

${ }^{2}$ To whom correspondence should be addressed.
}

The gross changes in response properties occur only during a limited time or "critical period" in normal kittens (Hubel and Wiesel, 1970; Cynader et al., 1980; Olson and Freeman, 1980; Levitt and Van Sluyters, 1982). Concentrations of endogenous NE are greater in the adult cortex than in the kitten cortex (Jonsson and Kasamatsu, 1983). Therefore, if NE is crucially involver in cortical plasticity, one would expect it to have a different effect upon neurons in kittens than it does in adults. The physiological evidence that exists to date shows the effect of NE or its absence most dramatically in changes in the properties of a population of neurons over periods of days. If the early stages of this mechanism involve changes in the postsynaptic membrane conductance or changes in the amount of presynaptic transmitter released, then these initial changes might be detected over a shorter period even though the final shift or its consolidation may take longer.

The most commonly suggested mechanism for the cortical changes occurring during visual deprivation involves competition between afferent terminals for control of cortical cells (Wiesel and Hubel, 1965; Guillery, 1972; Sherman et al., 1974; Hubel et al., 1977; Shatz and Stryker, 1978). A synaptic model for cortical plasticity has been proposed (Stent, 1973; Blakemore and Van Sluyters, 1975; Rauschecker and Singer, 1981) based on 
Hebb's (1949) neurophysiological postulate of learning. In general, this model proposes that when there is concurrent activity in presynaptic afferents and the postsynaptic cell, the synaptic connections between them are maintained or strengthened; whereas when the postsynaptic cell is active and the presynaptic terminals are silent, the synaptic connections are weakened.

From such a model there are several ways in which $\mathrm{NE}$ might act so as to promote the selective responsiveness of a neuron to a particular stimulus: (1) by reducing postsynaptic firing that is not concurrent with the stimulus, (2) by enhancing the postsynaptic firing that is concurrent with the stimulus, (3) by enhancing negative feedback onto afferent fibers not associated with the stimulus, or (4) by enhancing positive feedback onto afferent fibers associated with the stimulus. All of these actions might be thought of as either enhancing the strength of the "signal" (the firing of the postsynaptic neuron that is related to the particular stimulus) or reducing the "noise" (the firing that is unrelated to the stimulus). Stent (1973) proposed that immature synaptic receptors are unstable and easily dislodged from their positions in the membrane by sudden reversals in the local membrane potential. By shifting the activity of cells directly or by altering the activity of their presynaptic terminals, NE might promote the stability of certain synaptic connections which are initially dominant due to either a natural imbalance in afferent connections or an artificially induced imbalance, as is the case in visual deprivation. To assess such a model, it is necessary to observe the effects of NE on neuronal response properties in normal kittens.

Only a few studies have looked at the effects of NE on neuronal response properties of cells in the visual cortex. Reader (1978) looked at the responses to flashes of light in adult cats and found that NE iontophoresed near the recording electrode reduced the firing in $71 \%$ of the cells sampled, increased firing in only $1 \%$, and had no effect on the rest. Madar et al. (1980), using adult cats and rats, found that NE produced long-lasting inhibition of the spontaneous activity of most cells. In some cells, NE appeared to inhibit the spontaneous firing selectively. Kasamatsu and Heggelund (1982) used kittens which had been pretreated with 6-OHDA infused into the visual cortices for 1 week up until the time of recording, as well as one normal adult cat. They found that the firing of about a third of the sampled neurons was reduced by $\mathrm{NE}$, that of another third was enhanced, and the rest were not affected.

However, no study has looked at the effect of NE upon the response properties of neurons in normal kitten visual cortex and compared this with the effect in adult cats. If NE plays a significant role in promoting the changes in neuronal response properties that occur during visual deprivation, the model proposed by Stent suggests that NE might act on either presynaptic fibers or postsynaptic membrane conductances in such a way as to promote a stronger correlation between the activity in a cortical neuron and in its dominant presynaptic afferents. This should be reflected in changes in the strength of response to a stimulus in the presence of increased NE. It is also possible that NE might affect visual cortical neurons in kittens during the "critical period" differently from those in adults.

In the experiments described here, we studied the short-term effect of NE iontophoresed onto visual cortical neurons in normal kittens and adult cats. We searched for differences between the effect in kittens and adults, hoping that this would provide some clue to the mechanisms underlying the long-term changes in response properties that occur during visual deprivation.

\section{Materials and Methods}

Eleven kittens, aged 4 to 10 weeks, and 10 adult cats were anesthetized with halothane and paralyzed with pancuronium bromide (Pavulon, 0.6 to $1.5 \mathrm{mg} / \mathrm{hr}$ ). After completion of the surgery, anesthesia was maintained with $70 \% \quad \mathrm{~N}_{2} \mathrm{O}$ and $30 \% \quad \mathrm{O}_{2}$ with approximately $0.5 \%$ halothane. Artificial respiration was adjusted to maintain the expired $\mathrm{CO}_{2}$ concentration at 4 to $5 \%$. Body temperature was maintained at $37.5^{\circ} \mathrm{C}$ with a thermostatically controlled heating pad.

The skull and dura over the postlateral gyrus were opened, and the opening was filled with $3 \%$ agar in saline to minimize pulsations. This was covered with a thin layer of petroleum jelly (except where the electrode was inserted) to prevent drying of the agar and cortex.

Nictitating membranes were retracted with $10 \%$ phenylephrine $\mathrm{HCl}$ (Neosynephrine), and pupils were dilated with atropine sulfate. The eyes were refracted and focused on a tangent screen at 57 inches using neutral contact lenses of an appropriate curvature.

Extracellular recordings were made with either fiveor nine-barreled micropipettes with a tungsten wire inserted into one of the barrels for recording. The ninebarreled electrodes consisted of a central tungsten-inglass recording electrode with eight surrounding pipettes. Five-barreled electrodes consisted of five triangularshaped barrels in a pentagonal arrangement. Tips were beveled, and the barrel protruding the furthest contained the tungsten wire. The tips of both types of electrodes had diameters of 8 to $12 \mu \mathrm{m}$ at the base of the bevel. The tungsten wire protruded about 10 to $15 \mu \mathrm{m}$ and was coated with platinum black in some cases. The impedance of the recording electrode was typically 1 to 2 megohms at $1 \mathrm{kHz}$. Penetrations were made at an angle to the cortical surface and to depths of no more than 3 $\mathrm{mm}$ to minimize the possibility of damage by the flaring shaft of the multi-barreled electrodes.

L-Norepinephrine bitartrate or hydrochloride (Sigma, 0.2 or $0.3 \mathrm{M}, \mathrm{pH} 4$ ) was ejected with currents of 30 to 100 $\mathrm{nA}$ for periods of 1 to $4 \mathrm{~min}$. The lower currents were used for the initial test periods. For the majority of cells whose firing properties were altered by NE, currents of $50 \mathrm{nA}$ or less were effective. Altered firing was nearly always apparent within 1 to 2 min but often increased in magnitude with longer durations. A retaining current of $-10 \mathrm{nA}$ was used. Current and $\mathrm{pH}$ artifacts were usually tested for by passing current through another barrel containing a similar concentration of $\mathrm{NaCl}$ with $\mathrm{pH} 4$. For some cells, D-norepinephrine bitartrate (Adams, 0.2 or $0.3 \mathrm{M}, \mathrm{pH} 4$ ) was iontophoresed as a control. Current return was usually through another barrel containing 3 $\mathrm{M} \mathrm{NaCl}$. 
Neurons were isolated by moving a pattern of light stripes of various widths and orientations in front of the screen while advancing the microdrive. Action potentials were monitored on an oscilloscope, and pulses triggered by a window discriminator were sent to a computer to construct peristimulus histograms at regular (usually $30-$ sec) intervals before, during, and after each period of iontophoresis. Only neurons with action potentials for which clear isolation could be maintained throughout the recording period were analyzed.

Receptive fields of neurons were plotted with handmoved stimuli. Neurons with spatially separated $\mathrm{ON}$ and OFF-zones either to stationary, flashed stimuli or to moving bars were classified as simple cells, and those with mixed or overlapping $\mathrm{ON} / \mathrm{OFF}$ zones were classified as complex cells. After characterizing the optimal stimulus parameters for each neuron, NE was iontophoresed while stimulating the neuron repetitively by sweeping a bar of light across its receptive field at the most effective orientation and velocity.

Our criterion for an effect was a statistically significant change in the total number of spikes in a cell's response during the injection period when compared with the control period preceding it. In addition, the cell's response had to recover to the control level in order to be certain that the effect was not due to a general drift in the cell's responsiveness.

The ratio of visually evoked firing to spontaneous activity was calculated for cells which were spontaneously active in order to examine changes in the "signalto-noise" ratio.

\section{Results}

Data were collected on 60 well isolated neurons, 37 from kittens and 23 from adult cats. The responses of $72 \%$ (43 of 60 ) of all cells sampled were reduced by NE, while the responses of only a few cells ( 3 of 60 ) were increased. NE decreased the firing of approximately equal percentages of cells in both kittens $(70 \%, 26$ of 37$)$ and adults $(74 \%, 17$ of 23$)$. In $85 \%$ of the cells whose responses were affected by $\mathrm{NE}$, the effects were seen with currents of $50 \mathrm{nA}$ or less and were usually apparent within 1 to $2 \mathrm{~min}$ of current onset. Examples of the reduced activity of both simple and complex cells in kitten and adult visual cortex are illustrated in Figures 1 to 4 .

Figure 1 illustrates the response of a complex cell in a 4-week-old kitten. The peristimulus response histograms in Figure $1 A$ and $B$ show the total number of spikes during six trials taken during the 1-min periods at $A$ and $B$, respectively, in Figure $1 C$. Figure $1 A$ shows the response immediately prior to injecting $\mathrm{NE}$, and Figure $1 B$ shows the response during the final minute of NE injection. The response of the cell to both directions of movement is reduced, while still maintaining the slight preference for the first direction. Meanwhile, the very slight spontaneous activity of the cell is completely eliminated.

The histogram in Figure $1 C$ shows the changes in the response of this cell over a period of about $16 \mathrm{~min}$. Each bar represents the mean number of spikes in the cell's response to movement in the preferred direction. The cell's response was sampled at $30-$ sec intervals. NE (50 $\mathrm{nA}$ for $1.5 \mathrm{~min}$ ) reduced the response of this cell, while the same current passed through the $\mathrm{NaCl}$ barrel had no significant effect. When NE was injected over a longer time (presumably producing a greater concentration around the recorded neuron), the response was reduced even more.

Figure 2 illustrates the response of a simple cell in an 8 -week-old kitten. Figure $2 A$ shows the response of the cell immediately prior to $\mathrm{NE}$ injection, and Figure $2 B$ shows the response during the final minute of a 3-min injection. Both the response and the spontaneous activity of this cell decreased. Figure $2 C$ plots the mean number of spikes in the response to the preferred direction at 30sec intervals. This cell's activity decreased during each of the three NE injection periods. A more rapid reduction is seen in the last two periods when $80 \mathrm{nA}$ of current were used, as compared with $50 \mathrm{nA}$ during the first period. During two $\mathrm{NaCl}$ injection periods used to test for current artifacts, no significant change in the response occurred.

Figure 3 illustrates the response of a simple cell in an adult cat. The first two injections of L-NE produced a considerable reduction of the cell's response, while a similar injection of D-NE had no significant effect. Figure $3 A$ shows the response immediately prior to L-NE injection, and Figure $3 B$ shows the response during the final minute of a 4-min injection. The response in both directions decreased, while the relative strengths of the two directions of stimulation remained about the same. During the final NE injection period, twice as much current was passed. The recovery in this instance was much slower.

Figure 4 illustrates the response of a complex cell in an adult cat. Figure $4 A$ shows that this cell had a fairly large amount of spontaneous activity and a rather weak response. Figure $4 A$ and $B$ show the response immediately before and during $\mathrm{NE}$ injection. Both the response and the spontaneous activity decreased, and the cell remained bidirectional with a slight preference for the first direction. Figure $4 C$ shows the mean response for the first direction plotted at 30-sec intervals. Both NE injection periods produced a strong reduction of the response. The second period, in which a stronger current was passed, produced a much more rapid effect.

While most visual cortical cells have very little spontaneous activity, a few, such as this one, have enough spontaneous activity to compare the amount of reduction of the response to that of the spontaneous activity. The ratio of the response strength to the spontaneous activity is often referred to as a "signal-to-noise" ratio. We have calculated this ratio for 15 cells.

Figure 5 shows the result of this calculation for the cell in Figure 4. The upper histogram shows the mean response in the preferred direction at 30-sec intervals, the middle histogram shows the spontaneous activity for comparable periods of time, and the lower histogram shows the calculated signal-to-noise ratio. It is apparent that this ratio increases significantly during both $\mathrm{NE}$ injection periods.

Of 15 cells on which a similar analysis was carried out, 6 showed increases in their signal-to-noise ratios (3 of 

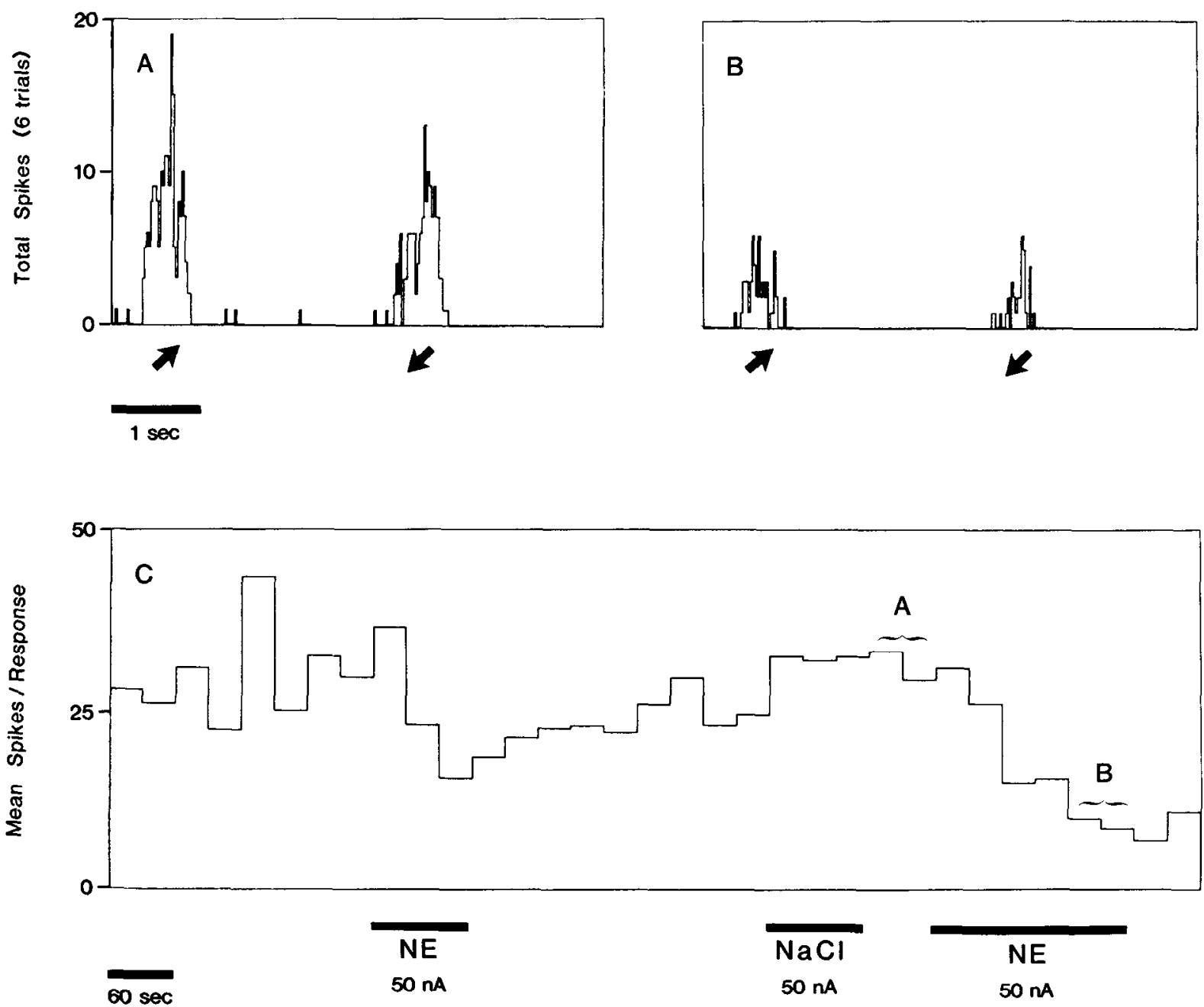

Figure 1. Reduced response during iontophoresis of NE on a complex cell in a 4-week-old kitten. $A$ and $B$ are peristimulus response histograms (bin width $=22 \mathrm{msec}$ ). A precedes a period of NE iontophoresis, whereas $B$ is from the final minute of a period of NE iontophoresis. The arrows below the histograms indicate the movement of a light bar in two directions. $C$ shows the mean number of spikes in the cell's response to movement in the preferred direction (the first direction for this bidirectional cell) taken from groups of three trials at 30 -sec intervals. The total spikes for the trials during the 1-min periods indicated by $A$ and $B$ in the lower histogram are illustrated in the upper two histograms. Periods of drug injection are indicated by the solid bars below the lower histogram.

which were statistically significant as judged from a twotailed $t$ test) and 4 showed decreases ( 3 of which were statistically significant). The others showed either no marked change or irregular changes over repeated trials. No difference was noticed between kittens and adults. In those cells which showed increases in signal-to-noise ratios, both the evoked and spontaneous firing were reduced, but the spontaneous firing was reduced to a greater extent.

Figure 6 summarizes our data. It shows that for the large majority of cells that were affected by $\mathrm{NE}$ the response decreased for both simple and complex cells in both kittens and adult cats. Overall, we found that $72 \%$ of the cells' responses decreased, whereas only about $5 \%$ were enhanced. Thus far we have noticed no difference in any of the effects of NE iontophoresed onto visual cortical cells of kittens when compared with those of adult cats.

\section{Discussion}

Since marked changes in the firing properties of visual cortical cells due to visual deprivation normally occur only in kittens and not in adult cats (Wiesel and Hubel, 1963; Hubel and Wiesel, 1970; Cynader et al., 1980; Olson and Freeman, 1980; Levitt and Van Sluyters, 1982), and since some evidence suggests that these changes depend upon the presence of NE (Kasamatsu and Pettigrew, 1976, 1979; Pettigrew and Kasamatsu, 1978; Kasamatsu et al., 1979; Bear et al., 1983; Daw et al., 1983a), it seemed possible that the behavior of visual cortical neurons in kittens in the presence of NE would differ in some way from that in adults. However, the data reported here show no difference between kittens and adults.

Only one previous study has investigated the effect of $\mathrm{NE}$ on visual cortical cells in kittens, that of Kasamatsu and Heggelund (1982). Their kittens had all been pre- 

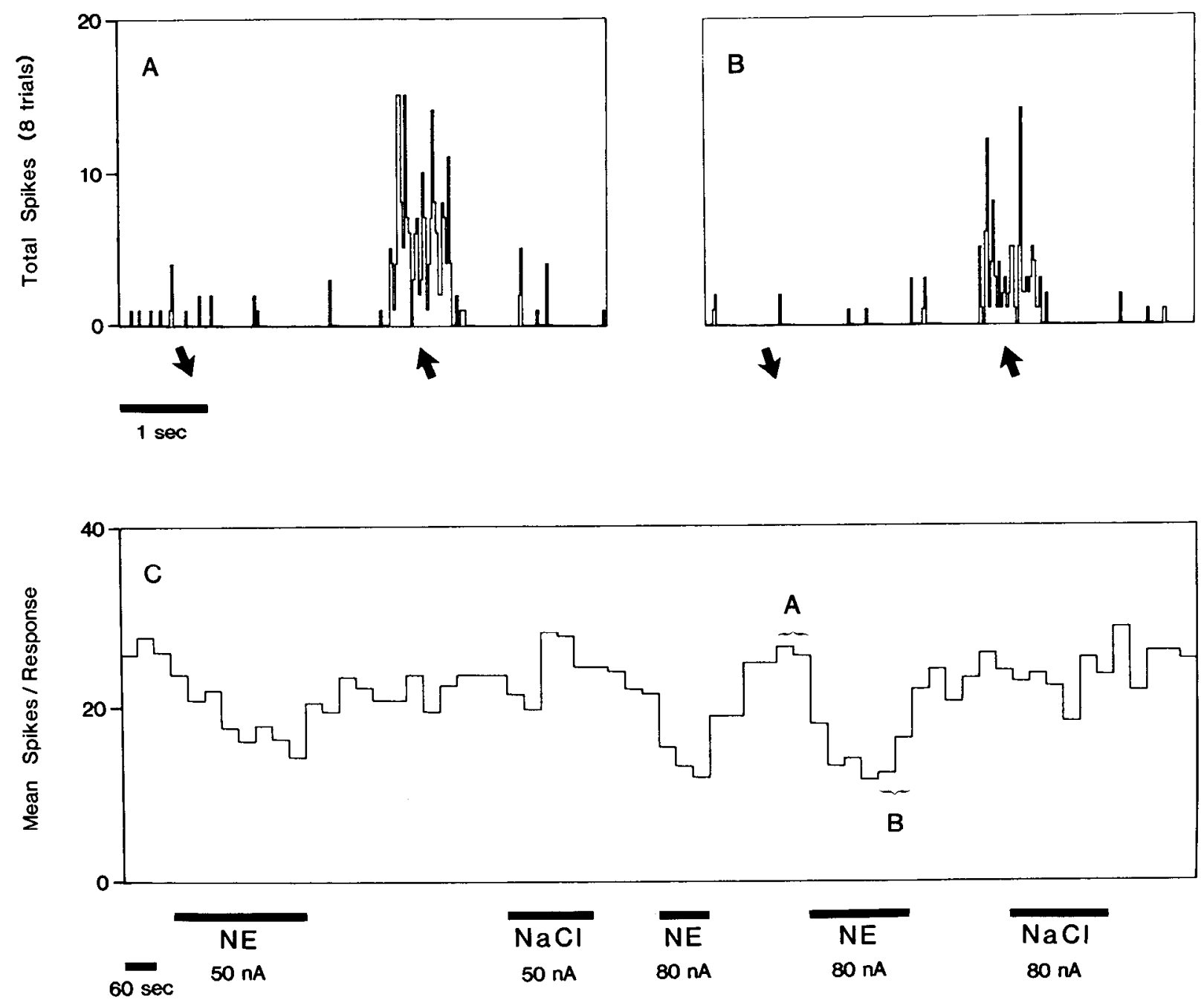

Figure 2. Reduced response during iontophoresis of NE on a simple cell in an 8-week-old kitten. See caption for Figure 1. Bin width $=22 \mathrm{msec}$ in $A$ and $B$.

treated with 6-OHDA intracortically starting 1 week before and continuing up to the time of recording and $\mathrm{NE}$ iontophoresis. This was intended to mimic as closely as possible the effect of restoring plasticity by infusion of NE into catecholamine-depleted cortices (e.g., Kasamatsu et al., 1979), so the animals differ from those reported here. Kasamatsu and Heggelund (1982) found that the firing of $36 \%$ of their sampled neurons was reduced by $\mathrm{NE}, 31 \%$ were enhanced, and $33 \%$ were not affected. This compares with our finding that the activity of $72 \%$ of the cells was reduced, $5 \%$ were enhanced, and $28 \%$ were not affected. Given the nature of single-unit recording while iontophoresing, it is possible that some differences could arise from either electrode sampling bias or from the location of the iontophoresed drug with respect to the recorded neuron. For example, it is possible that excitation could be due to iontophoresed NE suppressing inhibitory interneurons or, for that matter, that decreased activity could be due to exciting inhibitory interneurons (Hicks, 1983). While the electrode design differed in the two studies, the iontophoresing currents were in the same range.

There is some evidence that small concentrations of
NE may produce enhanced firing in some cortical cells while larger concentrations produce reduced activity in the same cells. Armstrong-James and Fox (1983) reported that about $6 \%$ of cells sampled in rat somatosensory cortex were excited by NE and that three-quarters of these were suppressed by higher concentrations (10 times or more) of NE. They also found that during long injections at constant currents the concentration of $\mathrm{NE}$ increases markedly with time. For two of the three cells which showed enhanced firing to NE in our study, this enhancement first increased and then decreased during a long NE injection ( 3 to $4 \mathrm{~min}$ ), possibly due to a reversal in the effect of $\mathrm{NE}$ at higher concentrations. However, none of the cells reported here whose firing was reduced showed an initial transient increase in firing. Moreover, the iontophoresing currents used in our study were in the same range as those of Kasamatsu and Heggelund (1982). Johnson et al. (1969) found that the proportion of cells in cat somatosensory cortex whose spontaneous firing was increased or decreased by NE varied with the type of anesthesia. Nitrous oxide was used in both our and Kasamatsu and Heggelund's (1982) studies, and we are not aware of any evidence that the small amounts of 
halothane we use to supplement the nitrous oxide would significantly alter the response properties we are studying. Thus, we are as yet uncertain whether the differences between the two studies is due to pretreatment with 6 OHDA, to electrode or experimenter sampling bias, or to some other factor.

A second difference in results is that we did not find a significant difference in the effectiveness of $\mathrm{NE}$ on simple versus complex cells. Whereas Kasamatsu and Heggelund (1982) reported that $86 \%$ of simple cells were affected by NE compared with only $33 \%$ of complex cells, we found that $68 \%$ (17 of 25 ) of simple cells and $79 \%$ (23 of 29) of complex cells were affected by NE.

Despite these differences, Kasamatsu and Heggelund (1982) appear to concur with our main finding, that there is no difference between the effect of NE iontophoresed onto visual cortical cells in kittens and adult cats. They reported "essentially similar results" in the effect of $\mathrm{NE}$ on cells in one normal adult cat compared with those in six 6-OHDA-pretreated kittens.

Several recent papers have emphasized the modulatory effect of NE. NE appears to enhance the evoked firing (and inhibition in some cases) of neurons with respect to their spontaneous or background firing for cells in the cerebellum (Moises et al., 1979; Woodward et al., 1979; Yeh et al., 1981; Yeh and Woodward, 1983), rat somatosensory cortex (Waterhouse and Woodward, 1980; Waterhouse et al., 1980), monkey auditory cortex (Foote et al., 1975), and in cat and rat visual cortex (Kasamatsu and Heggelund, 1982; Azizi et al., 1983). While our data do show some instances of enhanced evoked responses due to greater reduction of spontaneous activity, those instances comprise a relatively small proportion of our sample.

It should be remembered that an increase in the signalto-noise ratio might arise from any hyperpolarization of a neuron. A slight hyperpolarization would be expected to have a greater effect on the background discharge arising from synaptic potentials that are just above threshold for firing, and a lesser effect on evoked discharges arising from larger postsynaptic potentials. Such an effect might be elicited by any general inhibition of the cell. The argument for specific modulation can best be made when the evoked response of a neuron increases
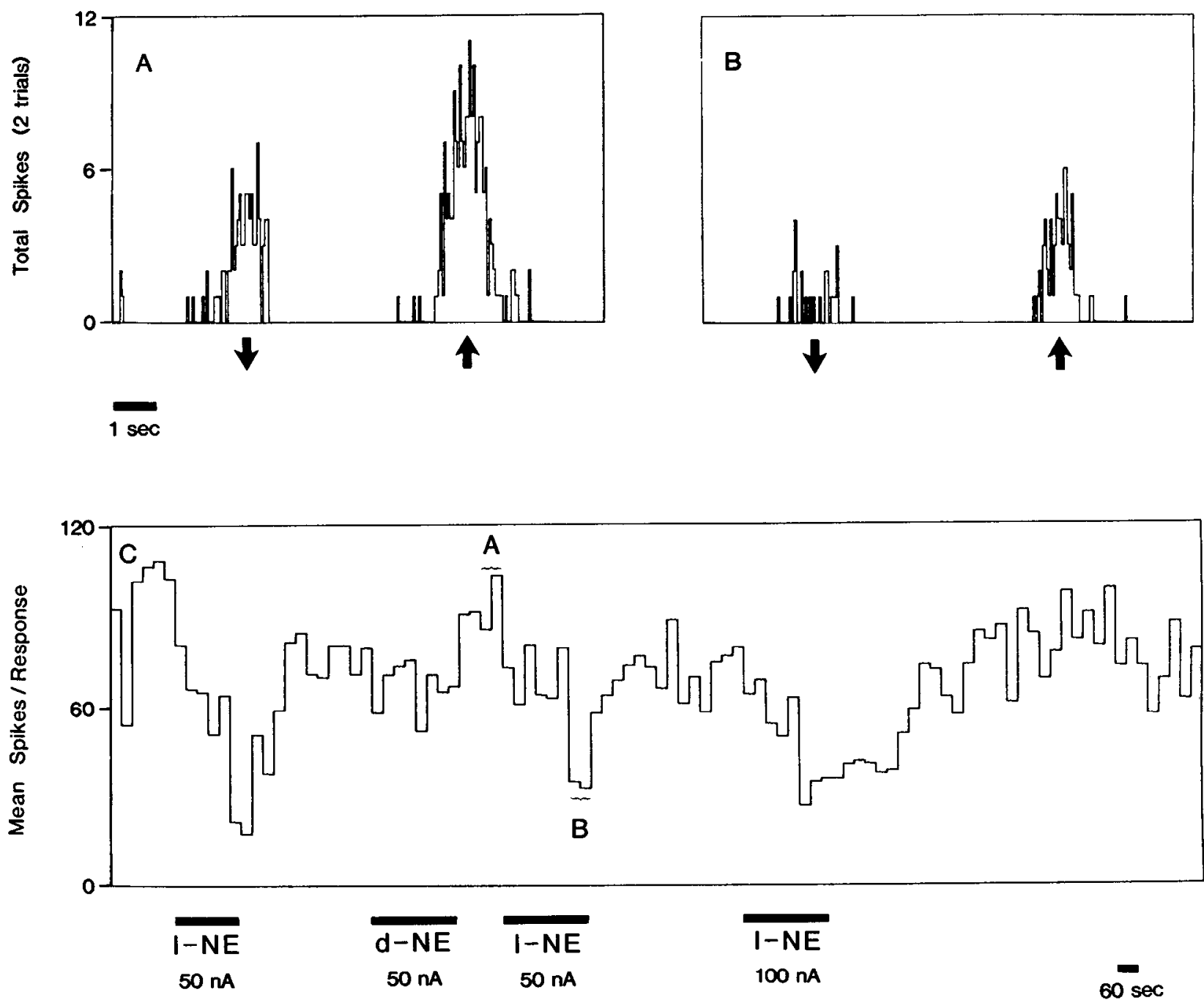

Figure 3. Reduced response during iontophoresis of NE on a simple cell in an adult cat. See caption for Figure 1. Bin width $=$ $44 \mathrm{msec}$ in $A$ and $B$. 

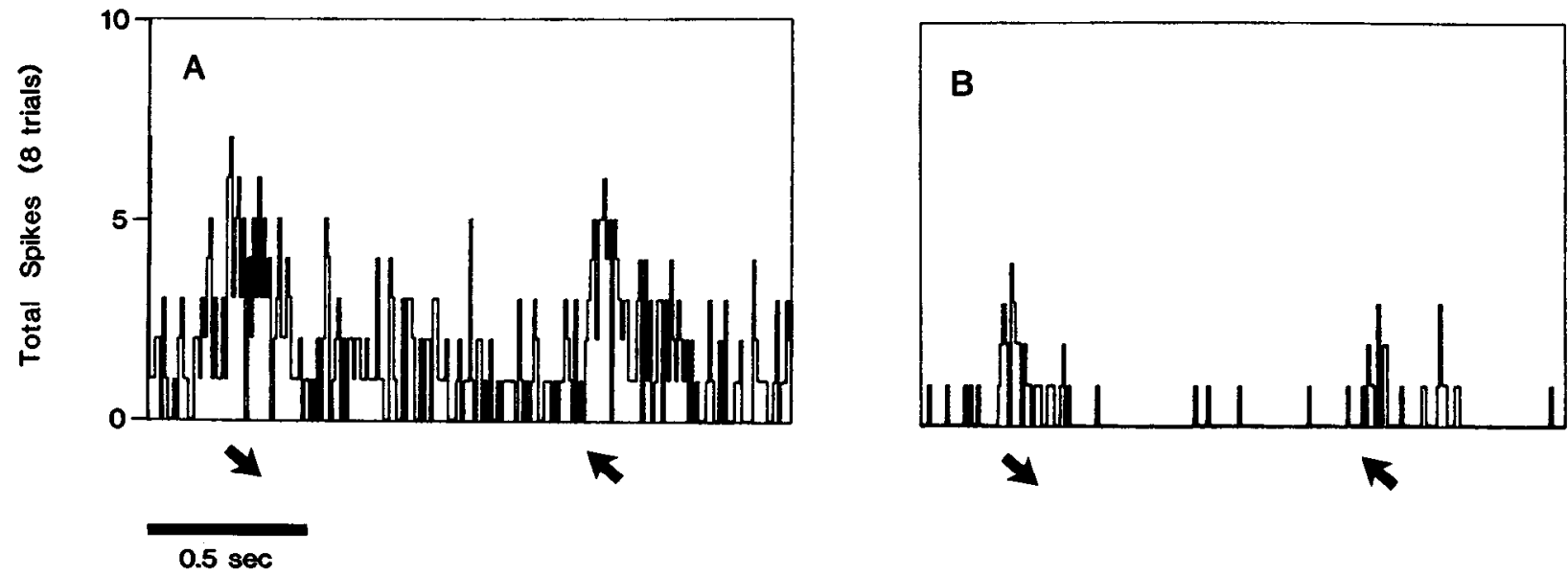

$0.5 \mathrm{sec}$

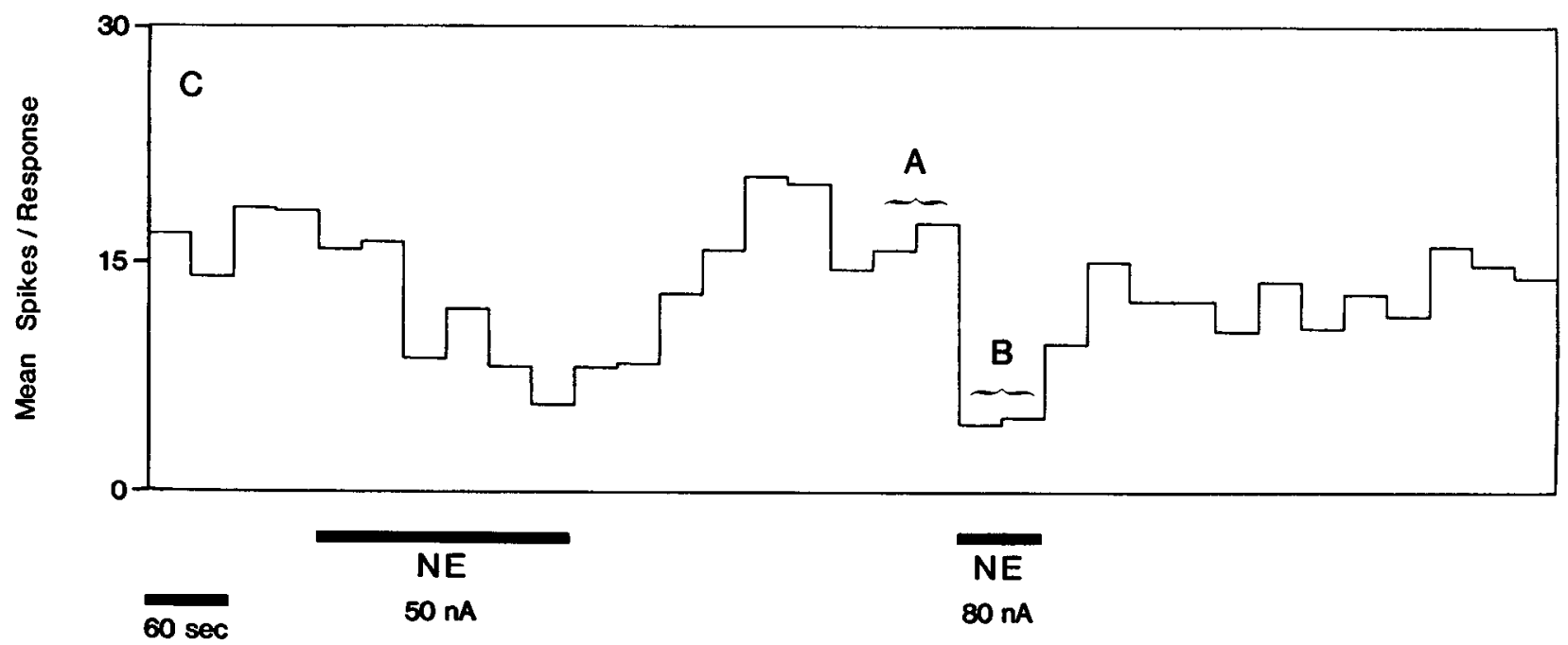

Figure 4. Reduced response during iontophoresis of NE on a complex cell in an adult cat. See caption for Figure 1. Bin width $=8 \mathrm{msec}$ in $A$ and $B$.

while the background discharge remains constant or decreases, or when the evoked response remains constant while the background discharge decreases.

In the majority of cortical cells in our sample, the spontaneous activity was very low, making meaningful comparisons of signal-to-noise ratios impossible. The nature of the problem can be seen in Figure 5 in a cell which showed significant and repeatable changes but which also shows the marked variability in the signalto-noise ratio that can arise from a small variability in the low spontaneous activity during NE injection.

Azizi et al. (1983) reported substantial proportions of neurons with enhanced evoked firing relative to spontaneous activity in rat visual cortex. It is interesting that their sample also contained a larger proportion of cells with significant amounts of spontaneous activity. Should $\mathrm{NE}$-induced enhancement prove to be common in cells with substantial spontaneous activity, it will be interesting to learn whether they represent a particular class of cells or whether they reflect more closely the normal behavior of cells in an alert animal.

The apparent general reduction of both the evoked and spontaneous firing of neurons by NE does not seem to support a model of plasticity which is based on synaptic receptors whose stability depends on local membrane potentials, as hypothesized by Stent (1973), and which also requires that $\mathrm{NE}$ is essential for promoting changes in response properties. While suppression of the neuron's firing reduces the occasions on which receptor molecules might be disrupted from the membrane, it would appear to do this for all stimuli, thus reducing the competitive interaction of different afferent terminals.

There are several possibilities for reconciling the observations that $\mathrm{NE}$ has no different effect on the evoked firing of visual cortical neurons in kittens than in adult cats and the observations that NE does not selectively enhance the responses of visual cortical neurons to an optimal stimulus with the hypothesis that NE plays an essential role in the plasticity evident in young animals reared with restricted visual input:

1. Possibly there are subtler effects that only become apparent when using stimuli nearer to the cells' threshold of firing. We have always chosen an optimal stimulus for eliciting an evoked response under the assumption that 
changes in firing would be most evident in the best response. Of course, it is possible that smaller changes in firing in the responses to near-threshold stimuli may have a greater influence on the development of a neuron's response properties, especially if they make the difference between a cell firing or failing to fire at all, than similar changes in the response to stimuli that evoke a strong response from the cell.
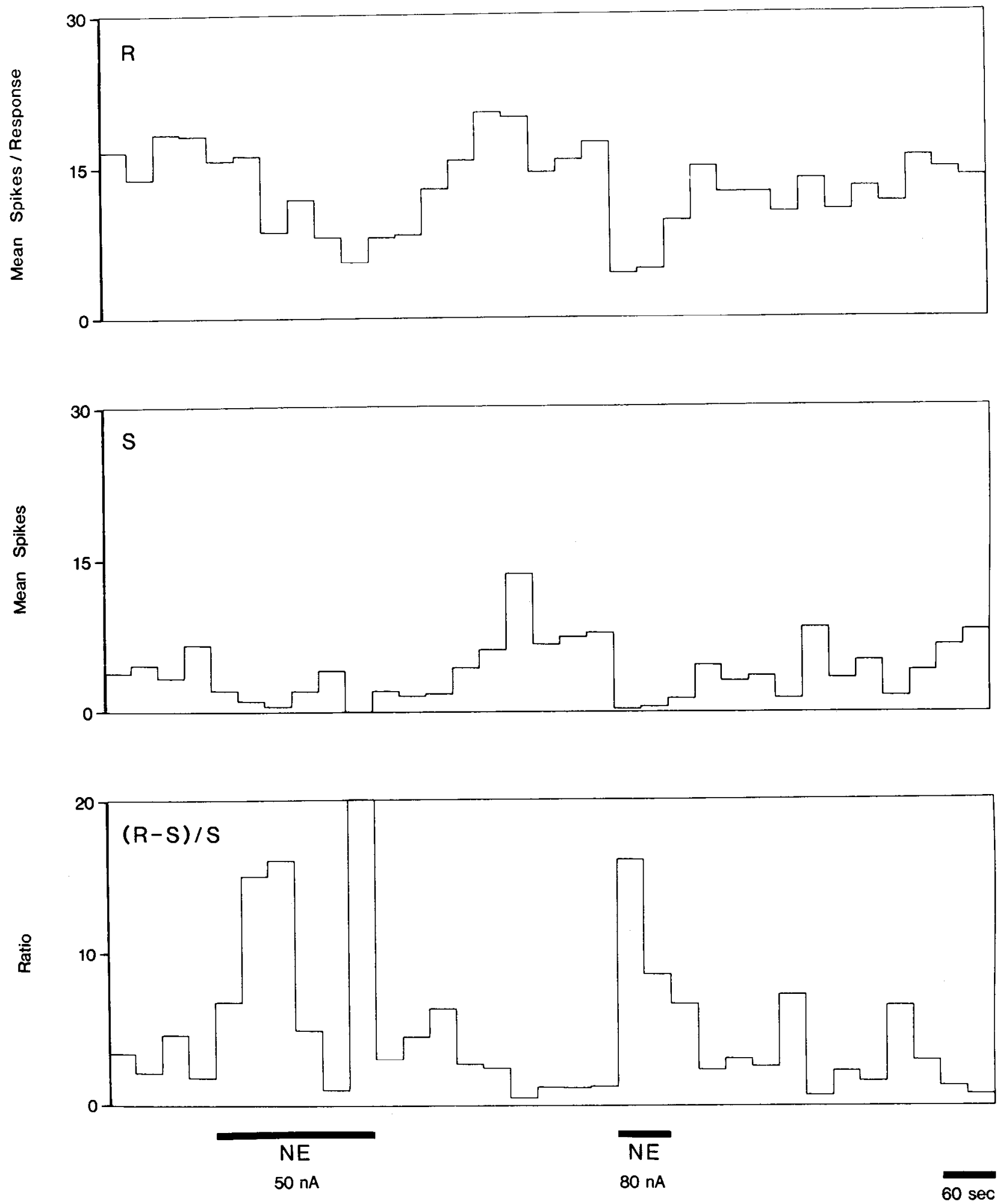

Figure 5. Signal-to-noise ratio for the complex cell represented in Figure 4. The upper histogram shows the mean number of spikes in the cell's response $(R)$ to movement in the preferred direction taken from groups of four trials at 30-sec intervals. The middle histogram shows the mean spontaneous activity $(S)$ during comparable times to those in the upper hislogram. The lower histogram is the calculated signal-to-noise ratio $((R-S) / S)$ for each 30 -sec interval. 


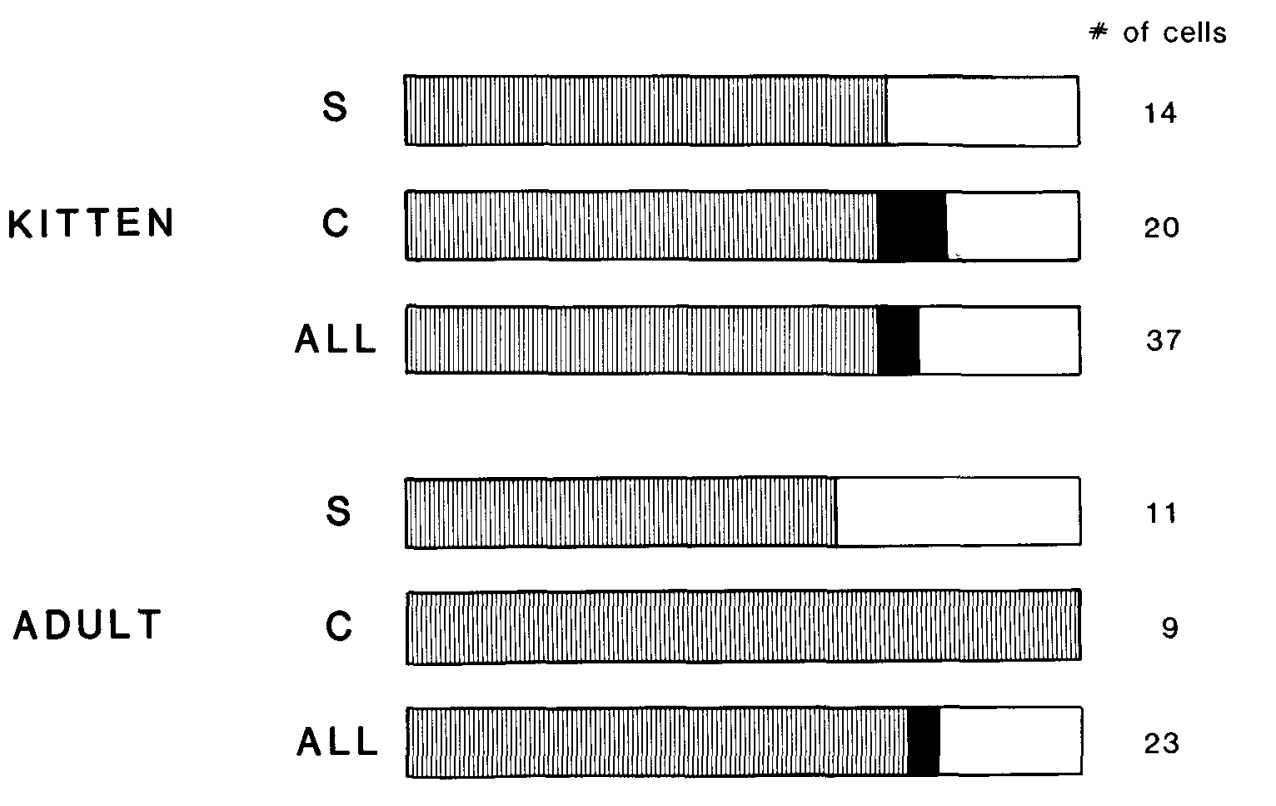

\section{COMBINED}

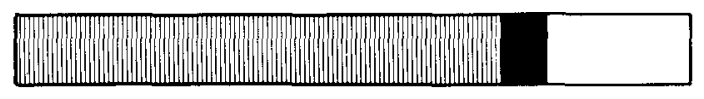

60
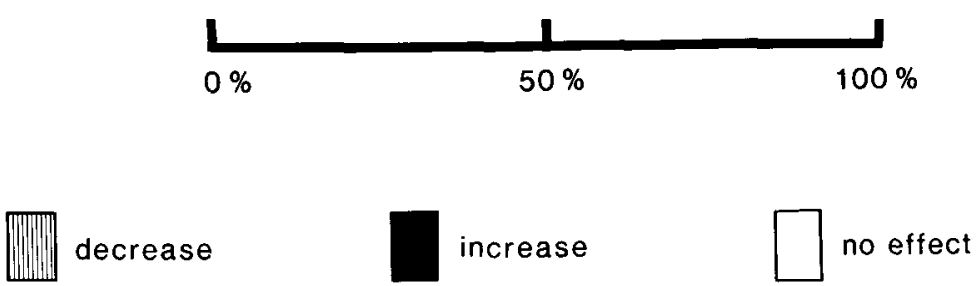

Figure 6. Percentages of cells whose activity was increased or decreased by NE. $S$ and $C$ stand for simple and complex cell types. The groups denoted by $A L L$ include some cells which were not classified as simple or complex.

2. It is also possible that the significant effect of NE is on processes which occur more slowly than those which affect the firing of the cell within a period of a few minutes. NE is thought to act on neurons by increasing the production of cAMP (summaries in Nathanson, 1977; Bloom, 1979; van Dongen, 1981), a substance which can in turn affect a variety of enzymatic processes within the cell. There is no reason to believe that only those effects of short enough duration to fall into the range easily studied by the electrophysiologist should be the significant ones for plasticity.

3. It is also possible that the changes in plasticity that occur when 6-OHDA is infused into the cortex are due to some effect besides the depletion of NE. Recent evidence has failed to support the original hypothesis that $\mathrm{NE}$ - is essential for the alteration of cortical neuronal response properties that occurs when young animals are reared in restricted visual environments (Adrien et al., 1982; Bear and Daniels, 1983; Daw et al., 1983b, 1984).

It may be that 6-OHDA causes aberrant activity in some population of cortical neurons and that the addition of NE suppresses this aberrant activity. Shaw and Cynader $(1981,1982,1984)$ were able to prevent the ocular dominance shift in monocularly deprived kittens by infusing glutamate into the visual cortex and by electrically stimulating the cortex. They suggested that an increase in spontaneous activity after NE depletion might prevent plasticity. If the predominant effect of $\mathrm{NE}$ on cortical neuronal activity is suppression, then the restoration of plasticity found by infusing $\mathrm{NE}$ into cortices depleted of NE by 6-OHDA (Kasamatsu and Pettigrew, 1979) might be due to a reduction of spontaneous activity or "noise" produced by 6-OHDA.

\section{References}

Adrien, J., P. Buisseret, Y. Frégnac, E. Gary-Bobo, M. Imbert, J. -P. Tassin, and Y. Trotter (1982) Noradrénaline et plasticité du cortex visuel du Chaton: un réexamen. C. R. Acad. Sci. Paris III 295: 745-750.

Armstrong-James, M., and K. Fox (1983) Effects of iontophoresed noradrenaline on the spontaneous activity of neurones in rat primary somatosensory cortex. J. Physiol. (Lond.) 335: 427-447.

Azizi, S. A., B. D. Waterhouse, R. A. Burne, and D. J. Woodward (1983) Modulatory actions of norepinephrine and serotonin on responses of simple and complex cells in rat visual 
cortex. Invest. Ophthalmol. Vis. Sci. 24 (Suppl.): 228.

Bear, M. F., and J. D. Daniels (1983) The plastic response to monocular deprivation persists in kitten visual cortex after chronic depletion of norepinephrine. J. Neurosci. 3: 407-416.

Bear, M. F., M. A. Paradiso, M. Schwartz, S. B. Nelson, K. M. Carnes, and J. D. Daniels (1983) Two methods of catecholamine depletion in kitten visual cortex yield different effects on plasticity. Nature 302: 245-247.

Blakemore, C., and R. C. Van Sluyters (1975) Innate and environmental factors in the development of the kitten's visual cortex. J. Physiol. (Lond.) 248: 663-716.

Bloom, F. E. (1979) Cyclic nucleotides in central synaptic function. Fed. Proc. 38: 2203-2207.

Cynader, M., B. Timney, and D. E. Mitchell (1980) Period of susceptibility of kitten visual cortex to the effects of monocular deprivation extends beyond six months of age. Brain Res. 191: 545-550.

Daw, N. W., R. K. Rader, T. W. Robertson, and M. Ariel (1983a) Effects of 6-hydroxydopamine on visual deprivation in the kitten striate cortex. J. Neurosci. 3: 904-914.

Daw, N. W., R. K. Rader, T. W. Robertson, and T. O. Videen (1983b) Do short term and long term depletion of noradrenaline have different effects on visual deprivation in the kitten visual cortex? Soc. Neurosci. Abstr. 9: 1217.

Daw, N. W., T. W. Robertson, R. K. Rader, T. O. Videen, and C. J. Coscia (1984) Substantial reduction of cortical noradrenaline by lesions of adrenergic pathway does not prevent effects of monocular deprivation. J. Neurosci. 4: 1354-1360.

Foote, S. L., R. Freedman, and A. P. Oliver (1975) Effects of putative neurotransmitters on neuronal activity in monkey auditory cortex. Brain Res. 86: 229-242.

Guillery, R. W. (1972) Binocular competition in the control of geniculate cell growth. J. Comp. Neurol. 144: 117-129.

Hebb, D. O. (1949) Organization of Behavior, John Wiley \& Sons, New York.

Hicks, T. P. (1983) Antagonism of synaptic transmission in vivo: Contributions of iontophoresis. Brain Behav. Evol. 22: $1-12$.

Hubel, D. H., and T. N. Wiesel (1970) The period of susceptibility to the effects of unilateral eye closure in kittens. J. Physiol. (Lond.) 206: 419-436.

Hubel, D. H., T. N. Wiesel, and S. LeVay (1977) Plasticity of ocular dominance columns in monkey striate cortex. Philos. Trans. R. Soc. Lond. Biol. 278: 377-409.

Johnson, E. S., M. H. T. Roberts, and D. W. Straughan (1969) The responses of cortical neurones to monoamines under differing anesthetic conditions. J. Physiol. (Lond.) 203: 261 280 .

Jonsson, G., and T. Kasamatsu (1983) Maturation of monoamine neurotransmitters and receptors in cat occipital cortex during postnatal critical period. Exp. Brain Res. 50: 449-458.

Kasamatsu, T., and P. Heggelund (1982) Single cell responses in cat visual cortex to visual stimulation during iontophoresis of noradrenaline. Exp. Brain Res. 45: 317-327.

Kasamatsu, T., and J. Pettigrew (1976) Depletion of brain catecholamines: Failure of ocular dominance shift after monocular occlusion in kittens. Science 194: 206-209.

Kasamatsu, T., and J. Pettigrew (1979) Preservation of binocularity after monocular deprivation in the striate cortex of kittens treated with 6-hydroxydopamine. J. Comp. Neurol. 185: $139-162$

Kasamatsu, T., J. Pettigrew, and M. Ary (1979) Restoration of visual cortical plasticity by local microperfusion of norepinephrine. J. Comp. Neurol. 185: 163-182.

Kasamatsu, T., J. Pettigrew, and M. Ary (1981) Cortical recovery from effect of monocular deprivation: Acceleration with norepinephrine and suppression with 6 -hydroxydopamine. J. Neurophysiol. 45: 254-266.
Levitt, F. B., and R. C. Van Sluyters (1982) The sensitive period for strabismus in the kitten. Dev. Brain Res. 3: 323327.

Madar, Y., M. Segal, and U. Kuhnt (1980) The functional role of the noradrenergic system in the visual cortex. Microiontophoretic study. Exp. Brain Res. 41: A14.

Moises, H. C., D. J. Woodward, B. J. Hoffer, and R. Freedman (1979) Interaction of norepinephrine with Purkinje cell responses to putative amino acid neurotransmitters applied by microiontophoresis. Exp. Neurol. 64: 493-515.

Movshon, J. A., and R. C. Van Sluyters (1981) Visual neural development. Annu. Rev. Psychol. 32: 477-522.

Nathanson, J. A. (1977) Cyclic nucleotides and nervous system function. Physiol. Rev. 57: 157-256.

Olson, C. R., and R. D. Freeman (1980) Profile of the sensitive period for monocular deprivation in kittens. Exp. Brain Res. 39: $17-21$.

Pettigrew, J. D., and T. Kasamatsu (1978) Local perfusion of noradrenaline maintains visual cortical plasticity. Nature 271: 761-763.

Rauschecker, J. P., and W. Singer (1981) The effects of early visual experience on the cat's visual cortex and their possible explanation by Hebb synapses. J. Physiol. (Lond.) 301: 215239.

Reader, T. A. (1978) The effects of dopamine, noradrenaline and serotonin in the visual cortex of the cat. Experientia 34: $1586-1588$

Shatz, C. J., and M. P. Stryker (1978) Ocular dominance in layer IV of the cat's visual cortex and the effects of monocular deprivation. J. Physiol. (Lond.) 281: 267-283.

Shaw, C., and M. Cynader (1981) Electrical or chemical stimulation of visual cortex prevents ocular dominance shift in monocularly deprived kittens. Soc. Neurosci. Abstr. 7: 772.

Shaw, C., and M. Cynader (1982) Evidence for post synaptic control of ocular dominance plasticity in kitten visual cortex. Neurosci Lett. Suppl. 10: S447-448.

Shaw, C., and M. Cynader (1984) Disruption of cortical activity prevents alterations of ocular dominance in monocularlydeprived kittens. Nature, in press.

Sherman, S. M., and P. D. Spear (1982) Organization of the visual pathways in normal and visually deprived cats. Physiol. Rev. 62: 738-855.

Sherman, S. M., R. W. Guillery, J. H. Kaas, and K. J. Sanderson (1974) Behavioral, electrophysiological and morphological studies of binocular competition in the development of the geniculo-cortical pathways of cats. J. Comp. Neurol. 158: 118.

Stent, G. S. (1973) A physiological mechanism for Hebb's postulate of learning. Proc. Natl. Acad. Sci. U. S. A. 70: 9971001.

Van Dongen, P. A. M. (1981) The central noradrenergic transmission and the locus coeruleus: A review of the data, and their implications for neurotransmission and neuromodulation. Prog. Neurobiol. 16: 117-143.

Waterhouse, B. D., and D. J. Woodward (1980) Interaction of norepinephrine with cerebrocortical activity evoked by stimulation of somatosensory afferent pathways in the rat. Exp. Neurol. 67: 11-34.

Waterhouse, B. D., H. C. Moises, and D. J. Woodward (1980) Noradrenergic modulation of somatosensory cortical neuronal responses to iontophoretically applied putative neurotransmitters. Exp. Neurol. 69: 30-49.

Wiesel, T. N., and D. H. Hubel (1963) Single cell responses in the striate cortex of kittens deprived of vision in one eye. J. Neurophysiol. 26: 1503-1517.

Wiesel, T. N., and D. H. Hubel (1965) Comparison of the effects of unilateral and bilateral eye closure on cortical unit responses in kittens. J. Neurophysiol. 28: 1029-1040. 
Woodward, D. J., H. C. Moises, B. D. Waterhouse, B. J. Hoffer, and R. Freeman (1979) Modulatory actions of norepinephrine in the central nervous system. Fed. Proc. 38: 2109-2116.

Yeh, II. II., and D. J. Woodward (1983) Noradrenergic action in the developing cerebellum: Interaction between norepinephrine and synaptically-evoked responses of immature
Purkinje cells. Dev. Brain Res. 11: 207-218.

Yeh, H. H., H. C. Moises, B. D. Waterhouse, and D. J. Woodward (1981) Modulatory interactions between norepinephrine and taurine, beta-alanine, gamma-aminobutyric acid and muscimol, applied iontophoretically to cerebellar Purkinje cells. Neuropharmacology 20: 549-560. 